\title{
DEFB4A is a potential prognostic biomarker for colorectal cancer
}

\author{
QIAN WU ${ }^{1,2^{*}}$, DAN WANG $^{1,2^{*}}$, ZHEN ZHANG $^{1,2}$, YAPING WANG ${ }^{1,2}$, \\ WEINA YU ${ }^{1,2}$, KAI SUN $^{1,2}$, NOMATHAMSANQA RESEGOFETSE MAIMELA ${ }^{1,2}$, \\ ZHENQIANG SUN ${ }^{3}$, JINBO LIU ${ }^{3}$, WEITANG YUAN ${ }^{3}$ and YI ZHANG ${ }^{1,2,4,5}$ \\ ${ }^{1}$ Biotherapy Center; ${ }^{2}$ Cancer Center; ${ }^{3}$ Department of Anorectal Surgery, The First Affiliated Hospital of Zhengzhou University; \\ ${ }^{4}$ School of Life Sciences, Zhengzhou University; ${ }^{5}$ Henan Key Laboratory for Tumor Immunology and \\ Biotherapy, Zhengzhou University, Zhengzhou, Henan 450052, P.R. China
}

Received January 9, 2020; Accepted July 8, 2020

DOI: 10.3892/ol.2020.11975

\begin{abstract}
Colorectal cancer (CRC) is the third leading cause of cancer-associated mortality. The present study aimed to investigate novel biomarkers to predict prognosis and provide a theoretical basis for studies of the pathogenesis and the development of therapies for CRC. The present study compared mRNA expression levels of patients with CRC with short- and long-term prognosis and of individuals with and without tumors in The Cancer Genome Atlas (TCGA) database. Differentially expressed genes (DEGs) were identified via volcano plot and Venn diagram analysis. Gene Ontology (GO) analysis and gene set enrichment analysis (GSEA) were performed to identify the functions of the DEGs, and the DEGs were further verified using clinical CRC samples. A total of 10 DEGs were identified as candidate genes using the TCGA database, and four DEGs [defensin $\beta$ 4A (DEFB4A), hyaluronan binding protein 2 (HABP2), oleoyl-ACP hydrolase and $\mathrm{TBC} 1$ domain family member $3 \mathrm{G}$ ] were associated with poor prognosis of patients with CRC. Two DEGs (DEFB4A and $H A B P 2$ ) were upregulated in tumor tissues of patients with CRC in the TCGA database. GO and GSEA analyses revealed that $D E F B 4 A$ was highly associated with immunosuppression, participates in 'myeloid leukocyte differentiation', 'leukocyte proliferation' and 'positive regulation of leukocyte-mediated immunity', and was positively correlated with CD11b, CD14, CD45, CD163 and IL17A. Furthermore, DEFB4A expression was significantly upregulated in patients with large tumors, advanced cancer stage, lymph node metastasis and liver metastasis. Survival analysis revealed that DEFB4A upregulation was associated with poor prognosis. DEFB4A
\end{abstract}

Correspondence to: Dr Yi Zhang, Biotherapy Center, The First Affiliated Hospital of Zhengzhou University, 1 Jianshe East Road, Zhengzhou, Henan 450052, P.R. China

E-mail: yizhang@zzu.edu.cn

${ }^{*}$ Contributed equally

Key words: defensin $\beta$ 4A, colorectal cancer, prognosis, immunity, biomarker gene knockdown experiments demonstrated that DEF4BA promotes cell migration. These results indicated that $D E F B 4 A$ potentially promotes tumor growth by regulating immunosuppressive activity and provided novel insights into the diagnosis and treatment of CRC.

\section{Introduction}

According to the Global Cancer Statistic of 2020, colorectal cancer (CRC) is the third most common (9\%) cancer and the second leading cause of cancer-associated mortality (9\%) worldwide (1). CRC was reported as the fourth most common and fatal cancer in China, in 2008 (2). Patients with CRC usually have a low survival rate and poor therapeutic responses, and are susceptible to progression and recurrence (3). Early diagnosis and effective treatment are critical to improve the survival of patients with CRC (4). CRC studies have focused on innovative ideas to identify molecular markers used to develop high-precision, non-invasive screening tests for CRC to increase population compliance and reduce the potentially harmful side effects associated with more invasive techniques (5). Diagnostic markers will give an indication of the likely progression of the disease (6). Targeting specific molecules in certain patients has facilitated more personalized treatments that help prevent or decelerate cancer progression. The present study aimed to determine prognostic factors and novel therapeutic targets to improve the survival of patients with CRC.

Previous studies have focused on the identification of molecules associated with tumor progression through genetic or mRNA profiling and screening of patients with colon cancer (7-12). For example, the expression profiles of long non-coding RNAs (lncRNAs) were compared at specific tumor stages (T0, T1, T2 and T3) in an azoxymethane/dextran sodium sulfate-induced primary colon cancer model and upregulation of the IncRNA H19 predicted a poor prognosis (7). Other studies analyzed microRNA (miR) expression profiles between tumor tissues and matched non-tumor tissues obtained from patients with CRC. For example, miR-124 is significantly downregulated in tumor tissues and associated with poor survival of patients with CRC, and may thus be considered to be a poor prognostic marker of CRC $(8,9)$. Furthermore, high expression levels of miR-203 
and miR-21 in serum are associated with poor survival of patients with CRC $(10,11)$. Analysis of The Cancer Genome Atlas (TCGA) database revealed that MMP19 is upregulated in patients with CRC and is associated with tumor progression (12). However, to the best of our knowledge, no study has directly screened mRNA profiles based on prognosis. The present study divided patients with CRC into different groups based on prognosis and screened the mRNA profiles of the respective groups.

Defensin $\beta$ 4A (DEFB4A), also known as $B D-2, S A P 1$, $D E F B 2, D E F B 4, H B D-2, D E F B-2$ and $D E F B 102$, belongs to the defensin family comprising cytotoxic peptides secreted by neutrophils, which serve important roles in innate immune defense against microbial infections (13-15). DEFB $4 A$ is upregulated in cutaneous squamous cell carcinoma and basal cell carcinoma $(16,17)$. It serves an important role in esophageal carcinogenesis both in vivo and in vitro (18). The genomic copy number of $D E F B 4 A$ has been analyzed in 466 patients with Crohn's disease and 329 controls, and an elevated $D E F B 4 A$ copy number has been identified as a risk factor for Crohn's disease regardless of disease origin (19). However, it remains unclear whether $D E F B 4 A$ expression is associated with the prognosis of CRC. Furthermore, the role of $D E F B 4 A$ in the immune system remains unclear.

The tumor microenvironment serves a significant role in tumor progression. Various immune elements comprise the tumor microenvironment, including bone marrow-derived cells, such as macrophages, $\mathrm{CD} 4^{+} \mathrm{T}$ cells, $\mathrm{CD} 8^{+} \mathrm{T}$ cells, B cells, natural killer cells and dendritic cells (20). Myeloid cells can differentiate into macrophages or myeloid-derived suppressor cells (MDSCs), which serve a tumorigenic role in the tumor microenvironment (21). MDSCs contribute to tumor vascular development by promoting angiogenesis and tumor growth (22). Tumor-associated macrophages (TAMs) are important regulators of tumorigenesis by inhibiting the antitumor effects of other cells, thus promoting tumor growth (23). However, it remains unclear whether $D E F B 4 A$ has a regulatory effect on the tumor microenvironment or whether it promotes CRC progression.

To identify candidate target genes that potentially prolong patient survival, mRNA expression profiles of tissues from CRC samples were compared in the TCGA database. Venn analysis was performed to determine candidate genes upregulated in tumor tissues among patients with poor prognosis. Subsequently, immune-associated pathway enrichment was analyzed using Gene Ontology (GO) and gene set enrichment analysis (GSEA), and the correlations between candidate target genes and certain immune cells were determined. Finally, clinical samples and CRC cell lines were obtained to verify the clinical significance of the identified genes. The present results may provide insights into targeted therapy for CRC.

\section{Materials and methods}

Acquisition of microarray data. Microarray data were obtained from TCGA (http://cancergenome.nih.gov/) (24). RNA-seq data for 784 samples were included in the dataset (Project ID: TCGA-COADREAD), including 689 tumor samples from patients with CRC and 95 normal tissues from healthy donors.
Identification of differentially expressed genes (DEGs). TCGA data were divided into two groups based on different categories: Patient prognosis and gene expression in tumor and normal tissues. Venn analysis of the two groups was performed, and 10 genes associated with CRC prognosis were identified.

Venn analysis. To identify candidate genes associated with patient survival, the gene expression profiles in the two groups were analyzed using the Venn Diagram web tool (http://bioinformatics.psb.ugent.be/webtools/Venn).

GO analysis. Functional analysis of the DEGs was performed using GO (http://www.geneontology.org) based on biological processes (25).

GSEA. GSEA was conducted using GSEA v4.0.3 software (https://www.gsea-msigdb.org/gsea/index.jsp) and the gene used in the present study was downloaded from the Molecular Signatures Database (MSigDB, http://software. broadinstitute. org/gsea/msigdb/index.jsp, v4.0). MSigDB curates various gene sets, including 1,320 canonical signaling pathways from BioCarta (https://cgap.nci.nih.gov/cgap_mitelman_retire_ notice.html), Kyoto Encyclopedia of Genes and Genomes (https://www.kegg.jp), PID (http://pid.nci.nih.gov), Reactome (https://reactome.org) and other pathway databases. TCGA data were analyzed via GSEA, and pathways with a false discovery rate $($ FDR $)<0.05$ were considered significant.

Patient characteristics. Tissue samples were obtained from 52 patients with CRC at The First Affiliated Hospital of Zhengzhou University (Zhengzhou, China) between April 2013 and April 2014. Patients underwent surgical resection or colonoscopy and the samples were verified via pathological analysis. The clinical characteristics of the patients are shown in Table I. A total of 34 men and 18 women were included in the present study. The median age was 60 years (age range, 26-91 years). CRC was diagnosed by two pathologists on the basis of pathological assessment. The collection of specimens was approved by the Institutional Ethics Committee of the First Affiliated Hospital of Zhengzhou University (Zhengzhou, China; approval no. Science-2010-LW-1213), and informed consent was obtained from each patient with available follow-up information.

Reverse transcription-quantitative PCR. Total RNA was extracted from 52 pairs of tumor and normal tissue samples from patients with CRC using TRIzol reagent (Invitrogen; Thermo Fisher Scientific, Inc.). Total RNA samples $(1 \mu \mathrm{g})$ were incubated at $42^{\circ} \mathrm{C}$ for $2 \mathrm{~min}$, followed by incubation at $37^{\circ} \mathrm{C}$ for $15 \mathrm{~min}$ and $85^{\circ} \mathrm{C}$ for $5 \mathrm{sec}$, according to the reverse transcription reaction protocol (Takara Biotechnology Co., Ltd.). The conditions of PCR were as follows: $95^{\circ} \mathrm{C} / 10 \mathrm{~min} ; 95^{\circ} \mathrm{C} / 10 \mathrm{sec}$, $60^{\circ} \mathrm{C} / 10 \mathrm{sec}, 72^{\circ} \mathrm{C} / 10 \mathrm{sec}, 40$ cycles (Premix Ex Taq II, Roche). Target gene expression was simultaneously assessed relative to that of GAPDH (a housekeeping gene and internal control). The following primers were used: DEFB4A forward, 5'-CTC CTCTTCTCGTTCCTCTTCA-3' and reverse, 5'-GCAGGT AACAGGATCGCCTAT-3'; and GAPDH forward, 5'-GGA GCGAGATCCCTCCAAAAT-3' and reverse, 5'-GGCTGTT 
Table I. Characteristics of patients with colorectal carcinoma.

\begin{tabular}{|c|c|c|}
\hline Characteristic & No. of cases & Percentage \\
\hline \multicolumn{3}{|l|}{ Sex } \\
\hline Male & 34 & 65.4 \\
\hline Female & 18 & 34.6 \\
\hline \multicolumn{3}{|l|}{ Age, years } \\
\hline$<60$ & 26 & 50.0 \\
\hline$\geq 60$ & 26 & 50.0 \\
\hline \multicolumn{3}{|l|}{ Treatment } \\
\hline Surgery & 42 & 80.7 \\
\hline Others & 10 & 19.3 \\
\hline \multicolumn{3}{|l|}{ Tumor size, mm } \\
\hline$<50$ & 28 & 53.8 \\
\hline$\geq 50$ & 24 & 46.2 \\
\hline \multicolumn{3}{|l|}{ Pathological type } \\
\hline Adenocarcinoma & 47 & 90.4 \\
\hline Others & 5 & 9.6 \\
\hline \multicolumn{3}{|c|}{ Lymph node metastasis } \\
\hline Yes & 19 & 36.5 \\
\hline No & 33 & 63.5 \\
\hline \multicolumn{3}{|l|}{ TNM stage } \\
\hline $\mathrm{I}$ & 12 & 23.1 \\
\hline II & 16 & 30.8 \\
\hline III & 16 & 30.8 \\
\hline IV & 8 & 15.3 \\
\hline \multicolumn{3}{|l|}{ Liver metastasis } \\
\hline Negative & 44 & 84.6 \\
\hline Positive & 8 & 15.4 \\
\hline \multicolumn{3}{|l|}{ Differentiation } \\
\hline Poor & 12 & 23.1 \\
\hline Medium-well & 40 & 76.9 \\
\hline
\end{tabular}

'Others' include chemotherapy and radiotherapy.

GTCATACTTCTCATGG-3'. The present study compared the expression levels of the target genes in clinical samples using the $2^{-\Delta \Delta \mathrm{Cq}}$ method (26). Expression levels of $D E F B 4 A$ and $G A P D H$ were examined for each sample and the relative expression levels of $D E F B 4 A$ were determined using the $2^{-\triangle \mathrm{Cq}}$ value of $D E F B 4 A$ divided by that of GAPDH (26).

Cell transfection. SW480 and HCT116 cells were seeded into a 24-well plate. SW480 and HCT116 cells were purchased from Chinese Academy of Sciences Cell Bank and cultivated with DMEM-high glucose containing 10\% FBS (Hyclone; GE Healthcare Life Sciences) and $1 \%$ penicillin-streptomycin, at $37^{\circ} \mathrm{C}$ in $5 \% \mathrm{CO}_{2}$. The growth status of the cells was closely observed until they reached a fusion rate of $80 \%$, and then cells were transfected with NC-small interfering RNA (negative control) (si-NC, sense: 5'-UUCUCCGAACGUGUC ACGUTT-3' and antisense: 5'-ACGUGACACGUUCGG AGAATT-3') and small interfering RNA targeting DEFB4A
(si-DEFB4A) (si-DEFB4A, sense: 5'-UCCUCUUCAUAUUCC UGAUTT-3' and antisense: 5'-AUCAGGAAUAUGAAGAGG ATT-3') purchased from Shanghai GenePharma Co., Ltd. with jetPRIME Polyplus transfection reagent (Polyplus-transfection SA). After $24 \mathrm{~h}$, the medium was changed to fresh medium, and cells were further incubated in $5 \% \mathrm{CO}_{2}$ for $48 \mathrm{~h}$. Subsequently, cells were collected for the subsequent experiments.

Wound healing assay. For the wound healing assay, SW480 and HCT116 cells were cultured in $500 \mu \mathrm{l}$ medium with $10 \%$ FBS (Hyclone) and the percentage of serum was in line with previous papers $(27,28)$. Sub-confluent tumor cells (80-90\%) were scraped using a sterile micropipette tip, and then serum-free medium was added. Next, cells were imaged at 0,12 and $24 \mathrm{~h}$ using an inverted fluorescence microscope (magnification, x200; Olympus Corporation).

Transwell assay. In the migration test, the transfected cells $\left(1 \times 10^{5}\right)$ were inoculated into the top chamber ( 8 microns) with $200 \mu \mathrm{l}$ serum-free medium. Complete medium $(600 \mu \mathrm{l})$ containing $10 \%$ FBS was added to the lower chamber (Corning, Inc.). Following incubation at $37^{\circ} \mathrm{C}$ for $24 \mathrm{~h}$, the migratory cells located under the insert were fixed and stained with Crystal Violet Staining Solution (Beyotime Institute of Biotechnology) at room temperature for $30 \mathrm{~min}$ and observed using an inverted fluorescence microscope (magnification, x200; Olympus Corporation).

Statistical analysis. The $\chi^{2}$ test was used to compare clinicopathological factors, and continuous variables were analyzed via unpaired Student's t-test or one-way ANOVA. Kaplan-Meier analysis and the log rank test were performed for survival analysis. Univariate and multivariate logistic regression models confirmed the associations between DEFB 4A expression and clinical features. Prism 7 (GraphPad Software, Inc.) was used for statistical analysis of all clinical samples. ANOVA was followed by Tukey's post-hoc test and performed using SPSS 16.0 for Windows (SPSS, Inc.). R software (version 3.4; R Foundation for Statistical Computing) was used for bioinformatics analysis. $\mathrm{P}<0.05$ was considered to indicate a statistically significant difference. All experiments were performed in triplicate and data are presented as the mean \pm standard deviation.

\section{Results}

Genes associated with poor prognosis. To identify genes associated with poor survival in the CRC cohort, patients were divided into two groups based on OS: Short ( $\leqq 1,000$ days) OS (patients with a survival time of 1,000 days would be included in short OS) and long (>1,000 days) OS (Fig. 1A). In total, 188 DEGs (fold change $>2$ ) were identified using a volcano plot, including 102 upregulated and 86 downregulated genes (Fig. 1B and C). Hierarchical cluster analysis revealed the expression profiles of the 188 DEGs (Fig. 1D). Subsequently, gene expression profiles in tumor and normal tissues (fold change $>2$ ) were analyzed, and it was observed that 916 genes were upregulated and 2,189 were downregulated in tumor tissues compared with in normal tissues $(\mathrm{P}<0.05$, FDR $<0.05$, fold change $>2$; Fig. $1 \mathrm{E}-\mathrm{G})$. The Venn diagram revealed that 
A

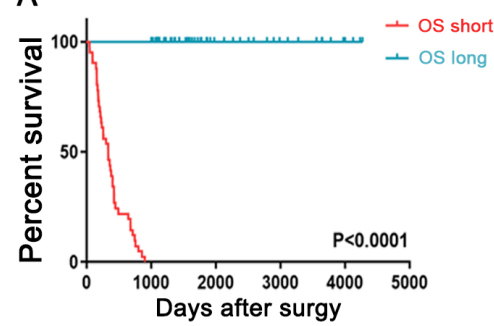

D

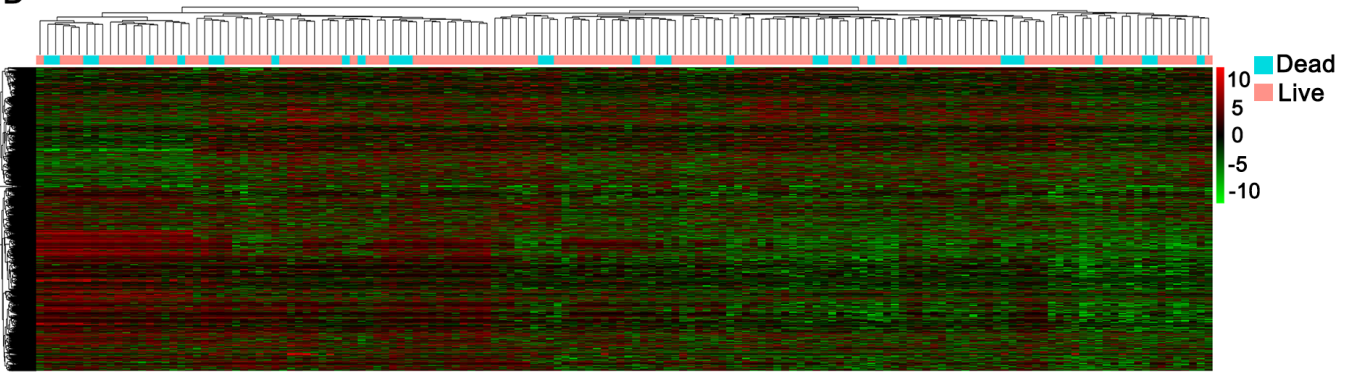

E

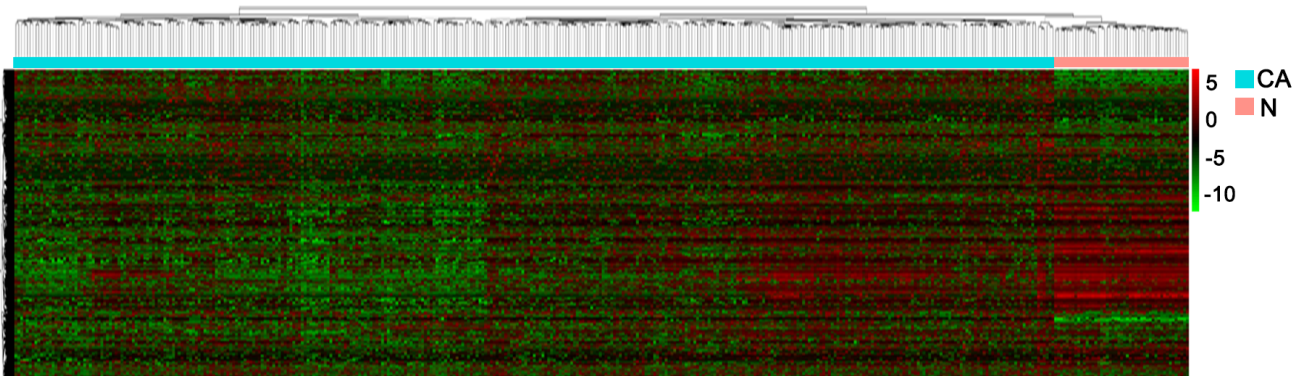

F

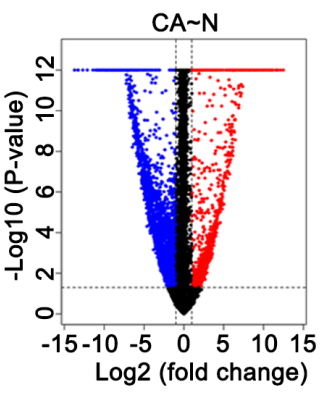

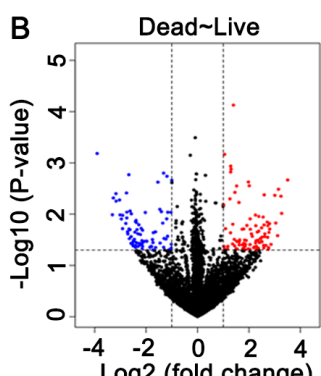

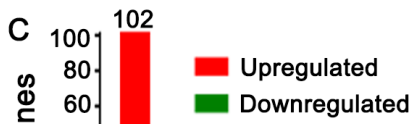

¿ 20

ฐั 40

.
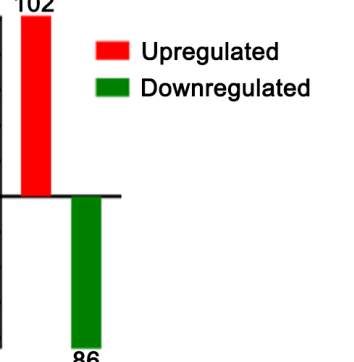
Table II. Upregulated genes $(\mathrm{n}=10)$ associated with a poor colorectal carcinoma prognosis in The Cancer Genome Atlas database.

\begin{tabular}{lrl}
\hline Gene symbol & Gene ID & \\
\hline DEFB4A & 1673 & Description \\
HABP2 & 3026 & Defensin beta 4A \\
OLAH & 55301 & Hyaluronan binding protein 2 \\
TBC1D3G & 101060321 & Oleoyl-ACP hydrolase \\
KISS1R & 84634 & TBC1 domain family member 3G \\
FRMD7 & 90167 & KISS1 receptor \\
SIO0A7A & 338324 & FERM domain containing 7 \\
OTX2 & 5015 & S100 calcium binding protein A7A \\
OR5M11 & 219487 & Orthodenticlehomeobox 2 \\
CHRNB3 & 1142 & Olfactory receptor family 5 subfamily M member 11 \\
\hline
\end{tabular}

performed. $D E F B 4 A$ was demonstrated to be involved in various biological processes (associated functional pathways are shown in Fig. 3A and B), and closely associated with 'myeloid leukocyte differentiation', 'leukocyte proliferation' and 'leukocyte mediated immunity', implying that DEFB4A potentially regulates the immune system. Finally, the database was searched for expression profiles of DEFB4A and immune-related genes, and a positive correlation between DEFB4A expression and the expression of immune markers, such as CD11b, CD14, CD45, CD163 and IL17A, was observed (Fig. 3C). These results suggest that DEFB4A is associated with poor prognosis in patients with CRC, potentially in an immunosuppressive myeloid leukocyte- and cytokine-dependent manner.

Validation in patient samples and clinical relevance of $D E F B 4 A$. To further clarify the clinical significance of $D E F B 4 A$ expression, the present study analyzed tissue samples from 52 patients with CRC. The associations between their mRNA expression levels and clinicopathological variables were observed. Detailed information of the patients is provided in Table III. DEFB4A expression was significantly upregulated in the CRC tumor tissues (Fig. 4A). Additionally, an association between $D E F B 4 A$ upregulation and advanced CRC stage (stage I, 12 cases; stage II, 16 cases; stage III, 16 cases; stage IV, 8 cases) and metastasis (M0, 44 cases; M1, 8 cases) was observed (Fig. 4B and C). Furthermore, DEFB4A upregulation in the tumor tissues was associated with poor prognosis ( $\mathrm{P}=0.0313$; Fig. 4D). Additionally, DEFB $4 A$ upregulation was significantly associated with advanced liver metastasis $(\mathrm{P}=0.039)$, stage $(\mathrm{P}=0.005)$, high $\mathrm{CA} 72-4$ value $(\mathrm{P}=0.003)$, tumor size $(\mathrm{P}=0.009)$ and lymph node metastasis ( $\mathrm{P}=0.044$; Table III). Therefore, DEFB4A was considered to be a prognostic marker associated with tumor progression in patients with CRC. Logistic regression analysis was performed to determine whether $D E F B 4 A$ can help predict the prognosis of CRC. Univariate analyses revealed that advanced TNM stage [odds ratio (OR), 8.00; $\mathrm{P}=0.01$ ], liver metastasis (OR, 4.21; $\mathrm{P}=0.03)$, lymph node metastasis (OR, 2.31; $\mathrm{P}=0.04)$, high CA199 level (OR,13.24; $\mathrm{P}=0.02)$, a high CA 72-4 level (OR, 10.19; $\mathrm{P}=0.01)$ and high DEFB4A level (OR, 2.15; $\mathrm{P}=0.02)$ were associated with the survival of patients with CRC. Furthermore, multivariate analyses revealed that advanced TNM stage (OR, 1.19; $\mathrm{P}=0.04)$, histological differentiation $(\mathrm{OR}, 0.67 ; \mathrm{P}<0.01)$, liver metastasis $(\mathrm{OR}, 3.62$; $\mathrm{P}=0.01)$, CA199 level (OR, 2.14; $\mathrm{P}=0.01)$, high CA 72-4 level $(\mathrm{OR}, 2.35 ; \mathrm{P}=0.05)$ and high DEFB4A level $(\mathrm{OR}, 1.45 ; \mathrm{P}=0.01)$ were independent prognostic predictors (Table IV). Overall, these results suggest that $D E F B 4 A$ serves an important role in predicting the prognosis of patients with CRC.

DEFB4A promotes proliferation and metastasis in CRC. To explore the biological roles of DEFB4A in CRC, DEFB4A expression was knocked down in HCT116 and SW480 cells (Fig. 5A and B). A wound healing assay revealed that DEFB4A knockdown inhibited the migration of HCT116 and SW480 cells (Fig. 5C). Transwell assays demonstrated that the migration of cells was decreased following knockdown of DEFB4A in SW480 cells compared with that in the NC group (Fig. 5D). The number of migratory cells decreased following knockdown of DEFB4A in SW480 cells (Fig. 5E). Overall, these results suggested that DEFB4A serves an important role in CRC development.

\section{Discussion}

With the increasing availability of high-throughput technologies, numerous novel biomarkers and therapeutic targets have been identified through transcriptomic analysis of various types of tumor. However, such studies on biomarkers in CRC have not been extensively performed. The identification of CRC biomarkers may help predict and prolong the survival of patients with CRC.

In the present study, mRNA profiling of microarray analysis data from the TCGA database was performed to identify numerous novel genes associated with poor prognosis in CRC. A critical role of $D E F B 4 A$ in patients with CRC was identified. The mRNA profiles of patients were first compared between the long OS and short OS groups, and between the tumor and normal tissue groups in the TCGA database. Subsequently, the present study investigated the association between mRNA expression and prognosis. $D E F B 4 A, H A B P 2, O L A H$ and $T B C 1 D 3 G$ were identified as potential predicators of poor prognosis. $D E F B 4 A$ and $H A B P 2$ 

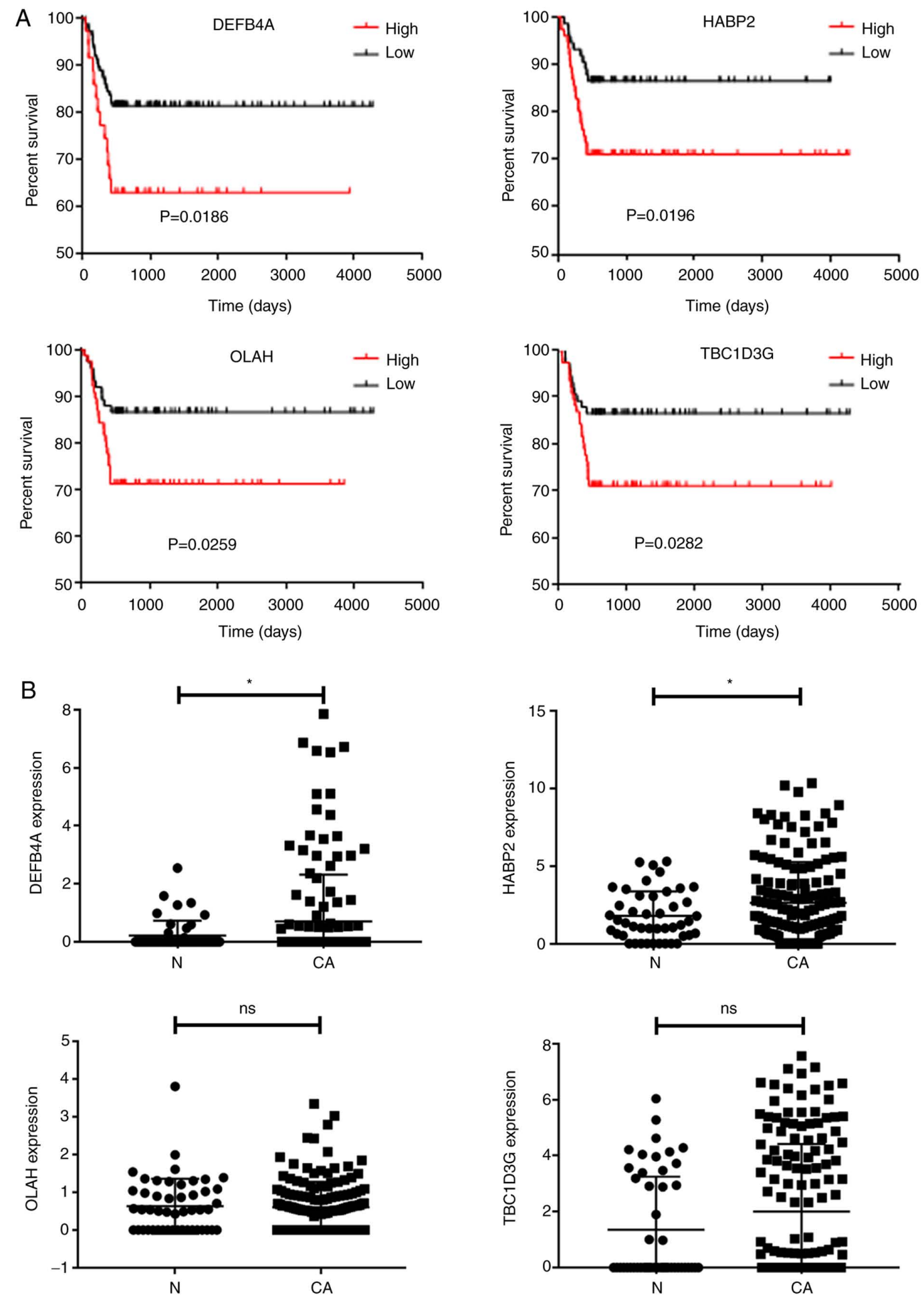

Figure 2. DEFB4A is upregulated based on data from TCGA and predicts poor prognosis. (A) Kaplan-Meier curve of four genes (DEFB4A, HABP2, OLAH and $T B C 1 D 3 G$ ) derived from data of patients included in the TCGA dataset. (B) mRNA expression levels of DEFB4A,HABP2,OLAH and TBC1D3G in cancer vs. control samples from patients in TCGA. " $\mathrm{P}<0.05$. ns, not significant; TCGA, The Cancer Genome Atlas; DEFB4A, defensin $\beta$ 4A; HABP2, hyaluronan binding protein 2; OLAH, oleoyl-ACP hydrolase; TBC1D3G, TBC1 domain family member 3G; CA, cancer; $\mathrm{N}$, normal.

were upregulated in CRC tissues of patients in the database. However, $H A B P 2$ has been reported to be downregulated in the sera of patients with CRC $(\mathrm{P}=0.0137)$ (29). Therefore, $D E F B 4 A$ was considered as a candidate gene for further analysis. GO and GSEA were used to assess the function of $D E F B 4 A$ in promoting disease progression and to highlight the role of $D E F B 4 A$ in the tumor microenvironment. DEFB $4 A$ was involved in 'myeloid leukocyte differentiation', 'leukocyte proliferation' and 'leukocyte mediated immunity'. Correlation analysis revealed that DEFB4A expression was positively correlated with immune markers, including CD11b, CD14, CD45, CD163 and IL17A. CD11b is expressed on the surface of a number of leukocytes, including monocytes, granulocytes and macrophages (30). CD14 is expressed on both monocytes 


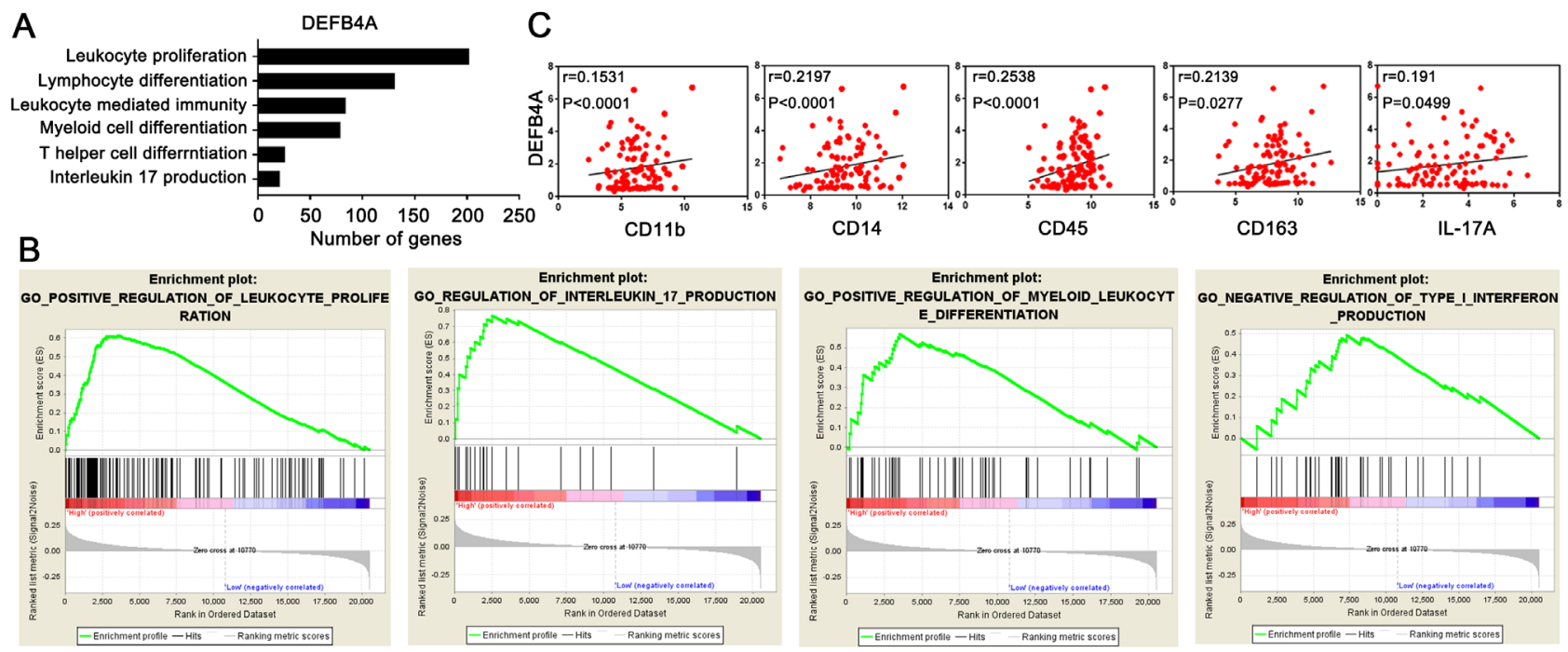

Figure 3. $D E F B 4 A$ is positively correlated with inhibitory immune cells. (A) Gene Ontology analysis revealed that $D E F B 4 A$ is involved in 'leukocyte proliferation', 'lymphocyte differentiation', 'leukocyte mediated immunity', 'myeloid cell differentiation', 'negative regulation of type I interferon production' and 'interleukin-17 production'. (B) Gene set enrichment analysis verified the results. (C) Correlation between DEFB4A and CD11b, CD14, CD45, CD163 and IL17A. $r$ and P-values are indicated. DEFB4A, defensin $\beta$ 4A; r, Pearson's correlation coefficient.

A

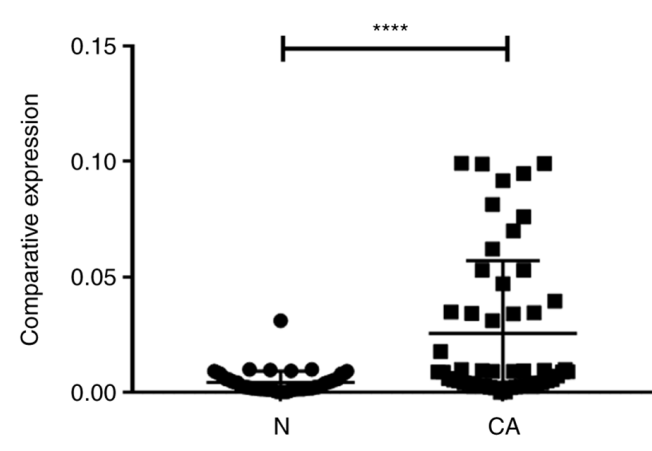

C

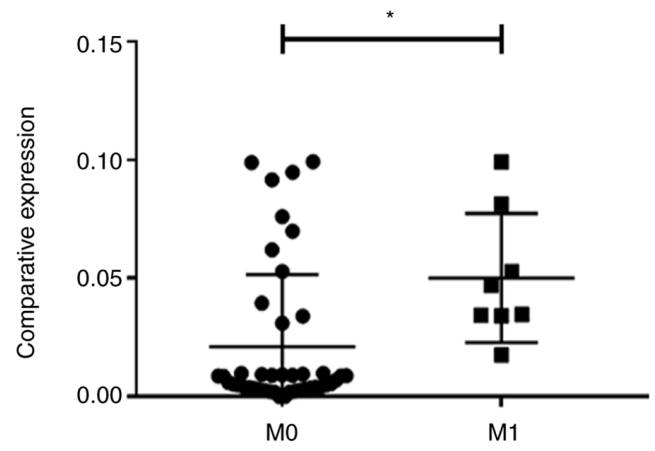

B

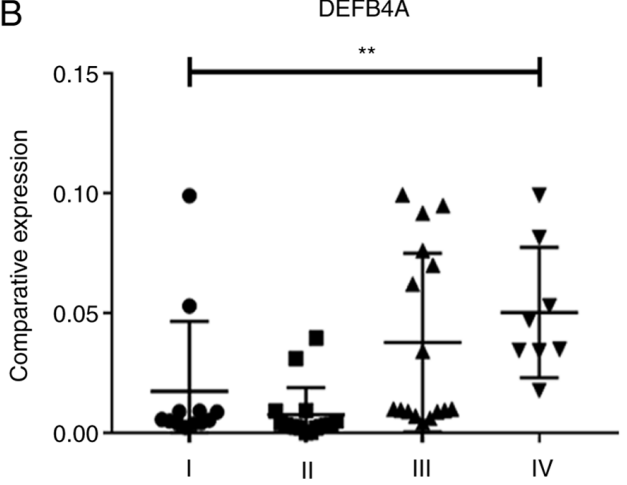

D

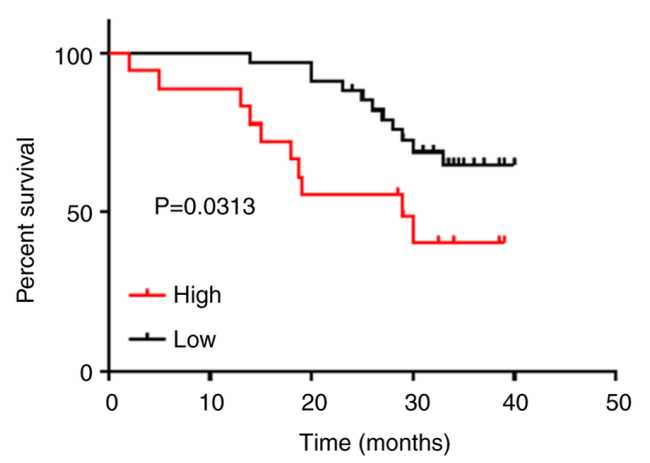

Figure 4. DEFB4A predicts poor prognosis in colorectal cancer. (A) DEFB4A mRNA expression in groups of cancer tissues and normal tissues are displayed. (B) DEFB4A mRNA levels were compared with respect to TNM stage. (C) DEFB4A mRNA expression levels of patients with different cancer stages. (D) Effect of DEFB4A expression on overall survival in patients with colorectal carcinoma $(\mathrm{n}=52)$. ${ }^{*} \mathrm{P}<0.05 ;{ }^{* * *} \mathrm{P}<0.01 ;{ }^{* * * * *} \mathrm{P}<0.0001$. DEFB4A, defensin $\beta$ 4A.

and macrophages, and CD45 is expressed on leukocytes. M2 macrophages may be marked with CD163 and M2 macrophages serve a role in promoting tumor growth (31). The OS of patients with non-small-cell lung cancer (32) and those with esophageal cancer $(33,34)$, with high M2 macrophage infiltration rates is shorter than those with low M2 macrophage infiltration rates. Patients with high expression levels of IL-17A had a poor prognosis in a CRC cohort (35). Previous studies have suggested that increased IL-17A promotes CRC in various animal models (36-38).

Analysis of clinical specimens of patients with CRC demonstrated that $D E F B 4 A$ expression was associated with 
Table III. Association between DEFB4A expression and clinicopathological characteristics of patients with colorectal carcinoma.

DEFB4A expression

\begin{tabular}{|c|c|c|c|c|c|}
\hline & & & & & \\
\hline Characteristic & Total, $\mathrm{n}$ & High, n & Low, $\mathrm{n}$ & $\chi^{2}$ & P-value \\
\hline Sex & & & & 0.000 & $>0.999$ \\
\hline Male & 34 & 17 & 17 & & \\
\hline Female & 18 & 9 & 9 & & \\
\hline Age, years & & & & 0.000 & $>0.999$ \\
\hline$<60$ & 26 & 11 & 15 & & \\
\hline$\geq 60$ & 26 & 15 & 11 & & \\
\hline Site of lesion & & & & 0.077 & 0.785 \\
\hline Colon & 21 & 13 & 8 & & \\
\hline Rectum & 31 & 13 & 18 & & \\
\hline Differentiation & & & & 1.194 & $0.330^{\mathrm{a}}$ \\
\hline Poor & 12 & 3 & 9 & & \\
\hline Well & 40 & 23 & 17 & & \\
\hline Tumor size, $\mathrm{cm}$ & & & & 7.212 & $0.009^{\mathrm{a}}$ \\
\hline$<5$ & 28 & 21 & 7 & & \\
\hline$\geq 5$ & 24 & 20 & 4 & & \\
\hline Pathological type & & & & 1.000 & $0.575^{\mathrm{a}}$ \\
\hline Adenocarcinoma & 47 & 25 & 22 & & \\
\hline Others & 5 & 3 & 2 & & \\
\hline Lymph node metastasis & & & & 4.064 & 0.044 \\
\hline No & 33 & 20 & 13 & & \\
\hline Yes & 19 & 13 & 6 & & \\
\hline Liver metastasis & & & & 5.005 & $0.039^{\mathrm{a}}$ \\
\hline No & 44 & 26 & 18 & & \\
\hline Yes & 8 & 8 & 0 & & \\
\hline Stage & & & & 8.026 & 0.005 \\
\hline $\mathrm{I} / \mathrm{II}$ & 28 & 18 & 10 & & \\
\hline III/IV & 24 & 18 & 6 & & \\
\hline CEA & & & & 0.001 & 0.974 \\
\hline Normal & 29 & 15 & 14 & & \\
\hline High & 23 & 11 & 12 & & \\
\hline CA 19-9 & & & & 3.315 & 0.139 \\
\hline Normal & 42 & 25 & 17 & & \\
\hline High & 10 & 1 & 9 & & \\
\hline CA 72-4 & & & & 5.678 & $0.003^{\mathrm{a}}$ \\
\hline Normal & 38 & 14 & 24 & & \\
\hline High & 14 & 2 & 12 & & \\
\hline
\end{tabular}

${ }^{\mathrm{a}}$ Fisher's exact test. All others were assessed using a $\chi^{2}$ test. DEFB4A, defensin $\beta 4 \mathrm{~A}$.

poor survival. Furthermore, $D E F B 4 A$ expression was upregulated in patients with CRC with advanced and metastatic cancer. Patients with CRC with high $D E F B 4 A$ expression had poor survival. In addition, knockdown of DEFB4A affected the migration ability of CRC cells.

TCGA data of patients with CRC were used to identify the DEGs between the long OS ( $>1,000$ days) and short OS ( $<1,000$ days) groups. In addition, mRNA expression was compared between tumor tissues and normal tissues in the same database. DEFB4A was highly expressed in tumors and associated with a poor prognosis. DEFB4A upregulation was associated with poor prognosis, and DEFB4A expression was significantly upregulated in patients with large tumors, advanced cancer stage, lymph node metastasis and liver metastasis. Another study used the Gene Expression Omnibus database to screen genes that are increased in patients with recurrence (39). Hierarchical clustering and pathway analyses revealed that thrombospondin 2 (THBS2) and cartilage 
Table IV. Logistic regression model analysis of liver metastasis predictors in patients with colorectal carcinoma.

\begin{tabular}{|c|c|c|c|c|c|c|}
\hline \multirow[b]{2}{*}{ Characteristics } & \multicolumn{3}{|c|}{ Univariate } & \multicolumn{3}{|c|}{ Multivariate } \\
\hline & OR & $95 \% \mathrm{CI}$ & P-value & OR & $95 \% \mathrm{CI}$ & P-value \\
\hline Sex (male vs. female) & 1.00 & $0.32-3.14$ & $>0.99$ & 0.78 & $0.06-10.56$ & 0.85 \\
\hline Age (<60 vs. $\geq 60$ years $)$ & 0.54 & $0.18-1.62$ & 0.27 & 13.05 & $0.93-183.71$ & 0.06 \\
\hline Tumor size $(<50$ vs. $\geq 50 \mathrm{~mm})$ & 1.00 & $0.34-2.98$ & $>0.99$ & 0.00 & $0.09-9.13$ & $>0.99$ \\
\hline Pathological type (adenocarcinoma vs. others) & 3.57 & $0.75-10.28$ & 0.06 & 2.45 & $0.61-2.76$ & 0.32 \\
\hline TNM stage (I/II vs. III/IV) & 8.00 & $2.42-16.81$ & 0.01 & 1.19 & $1.04-2.30$ & 0.04 \\
\hline Differentiation (medium vs. poor) & 0.34 & $0.10-1.18$ & 0.09 & 0.67 & $12.34-20.79$ & $<0.01$ \\
\hline Liver metastasis (no vs. yes) & 4.21 & $1.35-8.42$ & 0.03 & 3.62 & $1.24-7.78$ & 0.01 \\
\hline Lymph node metastasis (no vs. yes) & 2.31 & $1.24-3.56$ & 0.04 & 24.86 & $0.31-35.96$ & 0.92 \\
\hline $\mathrm{CEA}(<5$ vs. $\geq 5)$ & 1.17 & $0.39-3.50$ & 0.78 & 0.32 & $0.08-3.02$ & 0.78 \\
\hline CA199 (<35 vs. $\geq 35)$ & 13.24 & $1.53-114.30$ & 0.02 & 2.14 & $1.03-3.72$ & 0.01 \\
\hline CA724 (<6.9 vs. $\geq 6.9)$ & 10.19 & $2.00-52.80$ & 0.01 & 2.35 & $2.00-5.80$ & 0.05 \\
\hline DEFB4A (high vs. low) & 2.15 & $1.43-2.86$ & 0.02 & 1.45 & $1.02-1.89$ & 0.01 \\
\hline
\end{tabular}

OR, odds ratio.
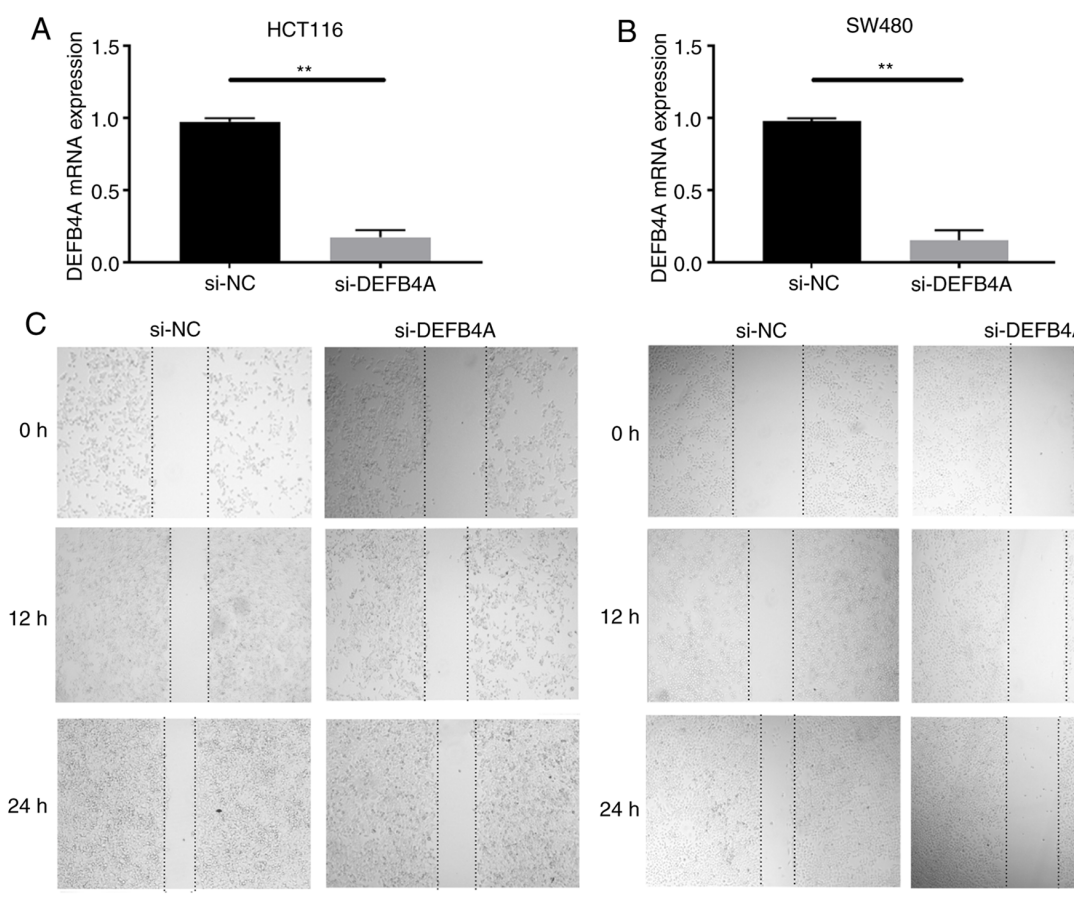

HCT116

$\mathrm{D}$
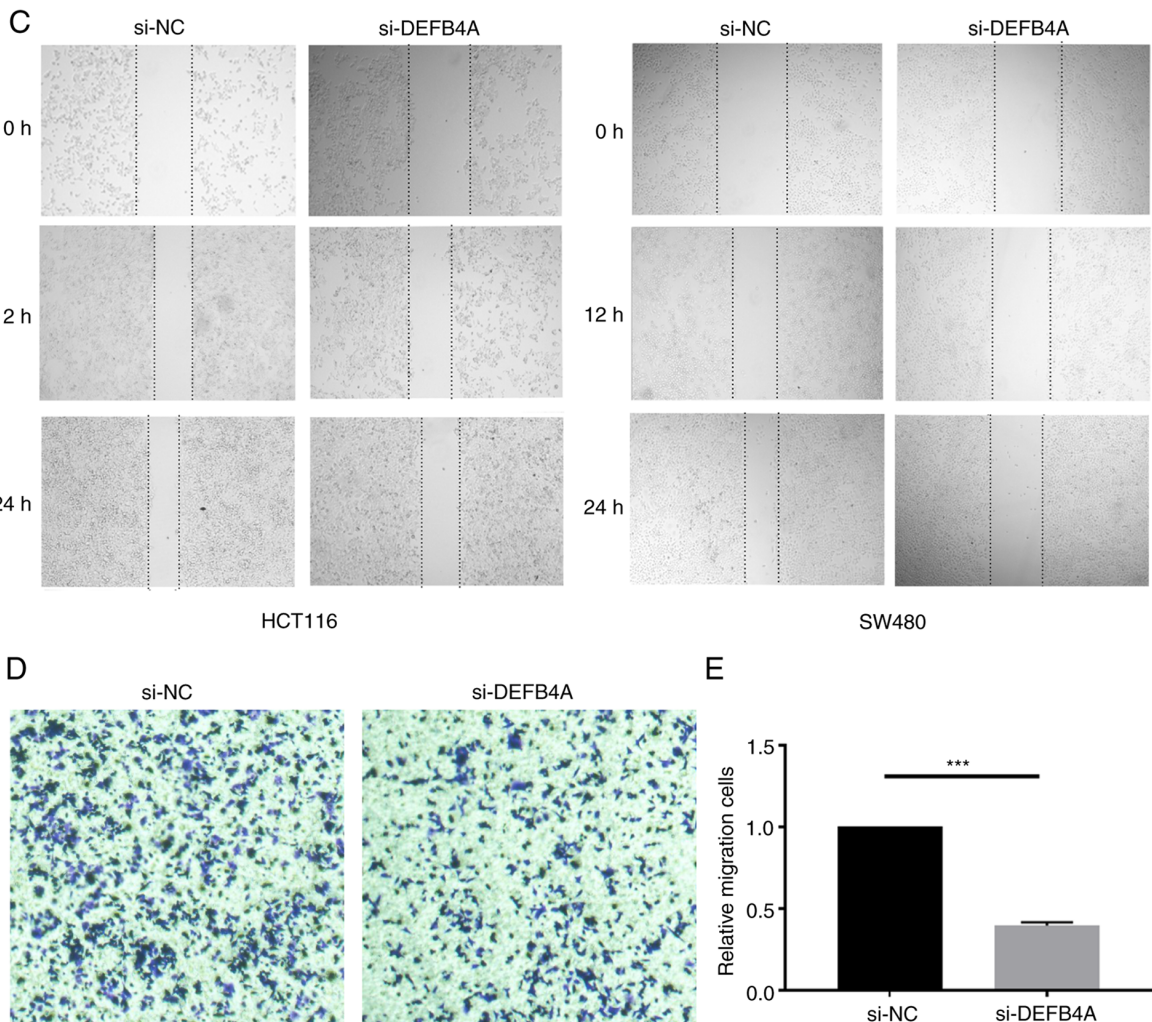

SW480

$\mathrm{E}$

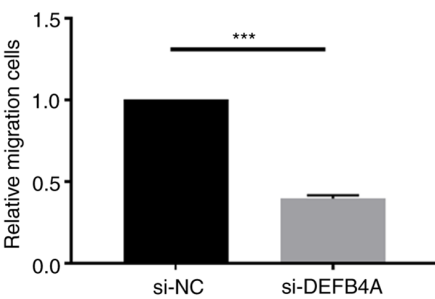

Figure 5. DEFB4A promotes colorectal cancer cell migration. (A) Expression levels of DEFB4A were detected using RT-qPCR following transfection of HCT116 cells with si-NC and si-DEFB4A. (B) Expression levels of DEFB4A were detected using RT-qPCR following transfection of SW480 cells with si-NC and si-DEFB4A. (C) Migration ability of cells was examined using a wounding healing assay. (D) Representative images were obtained for the Transwell assay (magnification, x200). (E) Proportions of migrated cells after $24 \mathrm{~h}$ were quantified. DEFB4A, defensin $\beta$ 4A; NC, negative control; RT-qPCR, reverse transcription-quantitative PCR; si, small interfering RNA. ${ }^{* *} \mathrm{P}<0.01 ;{ }^{* * *} \mathrm{P}<0.001$. 
oligomeric matrix protein (COMP) are associated with the ECM-receptor interaction, focal adhesion and TGF- $\beta$ signaling pathways (39). The hypergeometric distribution test demonstrated that the association between THBS2 and CRC is stronger than that of COMP (39). Pearson test results indicated that THBS2 might be considered to be a prognostic biomarker for CRC (39). To the best of our knowledge, this screening method and the hypothesis that DEFB4A may serve a pro-tumor role through immunosuppression have not been seen in other studies.

DEFB4A stimulates keratinocytes to release IL-18 and IL-20, pro-inflammatory cytokines serving as deciding factors in the pathogenesis of psoriasis (40). Furthermore, DEFB4A induction is required for Toll-like receptor (TLR) activation in monocytes through the convergence of IL-1 and vitamin $\mathrm{D}$ receptor signaling, and exerts direct bactericidal effects against $M$. tuberculosiss (41). The antimicrobial peptides DEFB4A and CAMP are inhibited by hsa-miR-21, leading to suppression of the TLR2/1-induced vitamin D antimicrobial signaling pathway (42). DEFB4A has been suggested as a biomarker for psoriasis because the clinical efficacy of targeted antibody therapy in psoriasis is associated with the inhibition of $D E F B 4 A$ expression (43). $D E F B 4 A$ expression can directly be inhibited by anthralin in vitro and in vivo, thus benefiting patients with psoriasis (44). However, it has remained unclear whether $D E F B 4 A$ is involved in the immunoregulation in CRC. GO analysis revealed that DEFB $4 A$ is involved in 'myeloid leukocyte differentiation', 'leukocyte proliferation' and 'positive regulation of leukocyte-mediated immunity'. Therefore, $D E F B 4 A$ may be associated with immunity in CRC.

To the best of our knowledge, the present study was the first to report $D E F B 4 A$ as a prognostic marker for CRC and as an immunoregulatory factor in the tumor microenvironment in patients with CRC. However, a limitation of the present study was that the research cohort was not large enough, which may affect the statistical results. In addition, the specific role of DEFB4A and immune factors in colon cancer and the underlying molecular mechanism need to be further explored.

In conclusion, to the best of our knowledge, $D E F B 4 A$ is upregulated in patients with CRC and is closely associated with poor prognosis. DEFB4A regulates immune function and potentially promotes immunosuppression. Therefore, $D E F B 4 A$ may be considered as a prognostic marker and immunotherapeutic target for CRC.

\section{Acknowledgements}

Not applicable.

\section{Funding}

The present study was supported by grants from the National Natural Science Foundation of China (grant nos. U1804281, 81771781 and 81602024) and funding from State's Key Project of Research and Development Plan (grant no. 2016YFC1303500).

\section{Availability of data and materials}

The datasets used and/or analyzed during the present study are available from the corresponding author on reasonable request.

\section{Authors' contributions}

YZ, QW and DW participated in the design and conception of the present study. YZ, QW, DW, ZS, JL and WTY were involved in data acquisition and analysis of certain clinical data. QW, DW, $\mathrm{ZZ}$ and YW performed the clinical experiments and analysis of the data. The manuscript was written by QW and critically reviewed by YZ, DW, ZZ, YW, WNY and NRM. WNY, KS and NRM were involved in performing and analyzing the cell experiments. All authors read and approved the final manuscript.

\section{Ethics approval and consent to participate}

The present study was approved by the Institutional Ethics Committee of the First Affiliated Hospital of Zhengzhou University (approval no. Science-2010-LW-1213), and informed consent was obtained from each patient with available follow-up information.

\section{Patient consent for publication}

Not applicable.

\section{Competing interests}

The authors declare that they have no competing interests.

\section{References}

1. Siegel RL, Miller KD and Jemal A: Cancer statistics, 2020. CA Cancer J Clin 70: 7-30, 2020.

2. Cidon EU: The challenge of metastatic colorectal cancer. Clin Med Insights Oncol 4: 55-60, 2010.

3. Vreeland TJ, Clifton GT, Herbert GS, Hale DF, Jackson DO, Berry JS and Peoples GE: Gaining ground on a cure through synergy: Combining checkpoint inhibitors with cancer vaccines. Expert Rev Clin Immuno 12: 1347-1357, 2016.

4. Siegel RL, Miller KD, Fedewa SA, Ahnen DJ, Meester RGS, Barzi A and Jemal A: Colorectal cancer statistics, 2017. CA Cancer J Clin 67: 177-193, 2017.

5. Dickinson BT, Kisiel J, Ahlquist DA and Grady WM: Molecular markers for colorectal cancer screening. Gut 64: 1485-1494, 2015.

6. Nikolouzakis TK, Vassilopoulou L, Fragkiadaki P, Mariolis Sapsakos T, Papadakis GZ, Spandidos DA, Tsatsakis AM and Tsiaoussis J: Improving diagnosis, prognosis and prediction by using biomarkers in CRC patients (Review). Oncol Rep 39: 2455-2472, 2018.

7. Dai L, Li J, Dong Z, Liu Y, Chen Y, Chen N, Cheng L, Fang C, Wang $\mathrm{H}$, Ji Y, et al: Temporal expression and functional analysis of long non-coding RNAs in colorectal cancer initiation. J Cell Mol Med 23: 4127-4138, 2019.

8. Qiu Z, Guo W, Wang Q, Chen Z, Huang S, Zhao F, Yao M, Zhao Y and He X: MicroRNA-124 reduces the pentose phosphate pathway and proliferation by targeting PRPS1 and RPIA mRNAs in human colorectal cancer cells. Gastroenterology 149: 1587-1598 e11, 2015.

9. Ma Y, Zhang P, Wang F, Zhang H, Yang J, Peng J, Liu W and Qin H: miR-150 as a potential biomarker associated with prognosis and therapeutic outcome in colorectal cancer. Gut 61: 1447-1453, 2012.

10. Toiyama Y, Takahashi M, Hur K, Nagasaka T, Tanaka K, Inoue Y, Kusunoki M, Boland CR and Goel A: Serum miR-21 as a diagnostic and prognostic biomarker in colorectal cancer. J Natl Cancer Inst. 105: 849-859, 2013.

11. Hur K, Toiyama Y, Okugawa Y, Ide S, Imaoka H, Boland CR and Goel A: Circulating microRNA-203 predicts prognosis and metastasis in human colorectal cancer. Gut 66: 654-665, 2017.

12. Chen Z, Wu G, Ye F, Chen G, Fan Q, Dong H, Zhu X and Wu C: High expression of MMP19 is associated with poor prognosis in patients with colorectal cancer. BMC Cancer 19: 448, 2019. 
13. Ganz T: Defensins: Antimicrobial peptides of innate immunity Nat Rev Immunol 3: 710-720, 2003.

14. Harder J, Bartels J, Christophers E and Schröder JM: A peptide antibiotic from human skin. Nature 387: 861, 1997.

15. Bajaj-Elliott M, Fedeli P, Smith GV, Domizio P, Maher L, Ali RS Quinn AG and Farthing MJ: Modulation of host antimicrobial peptide (beta-defensins 1 and 2) expression during gastritis Gut 51: 356-361, 2002.

16. Wei W, Chen Y, Xu J, Zhou Y, Bai X, Yang M and Zhu J: Identification of biomarker for cutaneous squamous cell carcinoma using microarray data analysis. J Cancer 9: 400-406, 2018

17. Gambichler T, Skrygan M, Huyn J, Bechara FG, Sand M, Altmeyer P and Kreuter A: Pattern of mRNA expression of beta-defensins in basal cell carcinoma. BMC Cancer 6: 163, 2006.

18. Shi N, Jin F, Zhang X, Clinton SK, Pan Z and Chen T: Overexpression of human $\beta$-defensin 2 promotes growth and invasion during esophageal carcinogenesis. Oncotarget 5: 11333-11344, 2014.

19. Bentley RW, Pearson J, Gearry RB, Barclay ML, McKinney C, Merriman TR and Roberts RL: Association of higher DEFB4 genomic copy number with Crohn's disease. Am J Gastroenterol 105: 354-359, 2010.

20. Joyce JA and Pollard JW: Microenvironmental regulation of metastasis. Nat Rev Cancer 9: 239-252, 2009.

21. Ortiz ML, Lu L, Ramachandran I and Gabrilovich DI: Myeloid-Derived Suppressor Cells in the Development of Lung Cancer 2: 50-58, 2014.

22. Yang L, DeBusk LM, Fukuda K, Fingleton B, Green-Jarvis B, Shyr Y, Matrisian LM, Carbone DP and Lin PC: Expansion of myeloid immune suppressor Gr+CD11b+ cells in tumor-bearing host directly promotes tumor angiogenesis. Cancer Cell 6 : 409-421, 2004.

23. Tacconi C, Ungaro F, Correale C, Arena V, Massimino L, Detmar M, Spinelli A, Carvello M, Mazzone M, Oliveira AI, et al: Activation of the VEGFC/VEGFR3 pathway induces tumor immune escape in colorectal cancer. Cancer Res 79: 4196-4210, 2019.

24. Cancer Genome Atlas Research Network: Comprehensive genomic characterization defines human glioblastoma genes and core pathways. Nature 455: 1061-1068, 2008

25. Gene Ontology Consortium: The Gene Ontology (GO) project in 2006. Nucleic Acids Res 34: D322-D326, 2006.

26. Livak KJ and Schmittgen TD: Analysis of relative gene expression data using real-time quantitative PCR and the 2(-Delta Delta C(T)) method. Methods 25: 402-408, 2001.

27. Liu Q, Cui X, Yu X, Bian BS, Qian F, Hu XG, Ji CD, Yang L, Ren Y, Cui W, et al: Cripto-1 acts as a functional marker of cancer stem-like cells and predicts prognosis of the patients in esophageal squamous cell carcinoma. Mol Cancer 16: 81, 2017.

28. Pan Z, Cai J, Lin J, Zhou H, Peng J, Liang J, Xia L, Yin Q, Zou B, Zheng J, et al: A novel protein encoded by circFNDC3B inhibits tumor progression and EMT through regulating Snail in colon cancer Mol Cancer 19: 71, 2020.

29. Brock R, Xiong B, Li L, Vanbogelen RA and Christman L: A multiplex serum protein assay for determining the probability of colorectal cancer. Am J Cancer Res 2: 598-605, 2012.

30. Solovjov DA, Pluskota E and Plow EF: Distinct roles for the alpha and beta subunits in the functions of integrin alphaMbeta2. J Biol Chem 280: 1336-1345, 2005.

31. Yang L and Zhang Y: Tumor-associated macrophages: From basic research to clinical application. J Hematol Oncol 10: 58, 2017.
32. Cao L, Che X, Qiu X, Li Z, Yang B, Wang S, Hou K, Fan Y, Qu X and Liu Y: M2 macrophage infiltration into tumor islets leads to poor prognosis in non-small-cell lung cancer. Cancer Manag Res 11: 6125-6138, 2019.

33. Li J, Xie Y, Wang X, Li F, Li S, Li M, Peng H, Yang L, Liu C, Pang L, et al: Prognostic impact of tumor-associated macrophage infiltration in esophageal cancer: A meta-analysis. Future Oncol 15: 2303-2317, 2019.

34. Yagi T, Baba Y, Okadome K, Kiyozumi Y, Hiyoshi Y, Ishimoto T, Iwatsuki M, Miyamoto Y, Yoshida N, Watanabe M, et al: Tumour-associated macrophages are associated with poor prognosis and programmed death ligand 1 expression in oesophageal cancer. Eur J Cance 111: 38-49, 2019.

35. Tosolini M, Kirilovsky A, Mlecnik B, Fredriksen T, Mauger S, Bindea G, Berger A, Bruneval P, Fridman WH, Pagès F and Galon J: Clinical impact of different classes of infiltrating t cytotoxic and helper cells (Th1, Th2, Treg, Th17) in Patients with Colorectal Cancer. Cancer Res 71: 1263-1271, 2011.

36. Huber S, Gagliani N, Zenewicz LA, Huber FJ, Bosurgi L, Hu B, Hedl M, Zhang W, O>Connor W Jr, Murphy AJ, et al: IL-22BP is regulated by the inflammasome and modulates tumorigenesis in the intestine. Nature 491: 259-263, 2012.

37. Grivennikov SI, Wang K, Mucida D, Stewart CA, Schnabl B, Jauch D, Taniguchi K, Yu GY, Osterreicher CH, Hung KE, et al: Adenoma-linked barrier defects and microbial products drive IL-23/IL-17-mediated tumour growth. Nature 491: 254-258, 2012.

38. Kirchberger S, Royston DJ, Boulard O, Thornton E, Franchini F, Szabady RL, Harrison O and Powrie F: Innate lymphoid cells sustain colon cancer through production of interleukin-22 in a mouse model. J Exp Med 210: 917-931, 2013.

39. Wang X, Zhang L, Li H, Sun WJ, Zhang H and Lai M: THBS2 is a potential prognostic biomarker in colorectal cancer. Sci Rep 6: 33366, 2016.

40. Hollox EJ, Huffmeier U, Zeeuwen PL, Palla R, Lascorz J, Rodijk-Olthuis D, van de Kerkhof PC, Traupe H, de Jongh G, den Heijer M, et al: Psoriasis is associated with increased beta-defensin genomic copy number. Nat Genet 40: 23-25, 2008.

41. Liu PT, Schenk M, Walker VP, Dempsey PW, Kanchanapoomi M, Wheelwright M, Vazirnia A, Zhang X, Steinmeyer A, Zügel U, et al: Convergence of IL-1beta and VDR activation pathways in human TLR2/1-induced antimicrobial responses. PLoS One 4: e5810, 2009.

42. Liu PT, Wheelwright M, Teles R, Komisopoulou E, Edfeldt K, Ferguson B, Mehta MD, Vazirnia A, Rea TH, Sarno EN, et al: MicroRNA-21 targets the vitamin D-dependent antimicrobial pathway in leprosy. Nat Med 18: 267-273, 2012.

43. Kolbinger F, Loesche C, Valentin MA, Jiang X, Cheng Y, Jarvis P, Peters T, Calonder C, Bruin G, Polus F, et al: $\beta$-Defensin 2 is a responsive biomarker of IL-17A-driven skin pathology in patients with psoriasis. J Allergy Clin Immunol 139: 923-932. e8, 2017.

44. Holstein J, Fehrenbacher B, Bruck J, Müller-Hermelink E, Schäfer I, Carevic M, Schittek B, Schaller M, Ghoreschi K and Eberle FC: Anthralin modulates the expression pattern of cytokeratins and antimicrobial peptides by psoriatic keratinocytes. J Dermatol Sci 87: 236-245, 2017.

This work is licensed under a Creative Commons Attribution-NonCommercial-NoDerivatives 4.0 International (CC BY-NC-ND 4.0) License. 